\title{
I-Vague Vector Spaces
}

\author{
Zelalem Teshome Wale
}

\begin{abstract}
The notions of I-vague vector spaces of vector spaces with membership and non-membership functions taking values in an involutary dually residuated lattice ordered semigroup are introduced which generalizes the notions with truth values in a Boolean algebra as well as those usual vague sets whose membership and non-membership functions taking values in the unit interval $[0,1]$. We discuss some properties of I-vague vector spaces.
\end{abstract}

Index Terms-Involutary dually residuated lattice ordered semigroup, I-vague sets, I-vague vector spaces.

\section{INTRODUCTION}

$\mathbf{R}$ AMAKRISHNA and Eswarlal [1] studied Boolean vague sets where the vague set of the universe $X$ is defined by the pair of functions $\left(t_{A}, f_{A}\right)$ where $t_{A}$ and $f_{A}$ are mappings from a set $X$ into a Boolean algebra satisfying the condition $t_{A}(x) \leq f_{A}(x)^{\prime}$ for all $x \in X$ where $f_{A}(x)^{\prime}$ is the complement of $f_{A}(x)$ in the Boolean algebra. K.L.N Swamy [2], [3], [4] introduced the concept of a Dually Residuated Lattice Ordered Semigroup (in short DRL-semigroup) which is a common abstraction of Boolean algebras and lattice ordered groups. The subclass of DRL-semigroups which are bounded and involutary (i.e. having 0 as the least, 1 as the greatest and satisfying $1-(1-x)=x)$ which is categorically equivalent to the class of MV-algebras of Chang [5] and well studied offer a natural generalization of the closed unit interval $[0,1]$ of real numbers as well as Boolean algebras. Thus, the study of vague sets $\left(t_{A}, f_{A}\right)$ with values in an involutary DRL-semigroup promises a unified study of real valued vague sets and also those Boolean valued vague sets.

T. Eswarlal and N. Ramakrishna [6] studied vague fields and vector spaces. Moreover, K.V. Rama Rao and Amarendra Babu V. [7] studied vague vector spaces and vague Modules. In this paper, using the definition of I-vague sets in [8], we defined and studied I-vague vector spaces where I is an involutary DRL-semigroup which generalizes the work of vector spaces discussed in T. Eswarlal and N. Ramakrishna [6] and K.V. Rama Rao and Amarendra Babu V. [7].

\section{Preliminaries}

Definition 1: A system $A=(A,+, \leq,-)$ is called a dually residuated lattice ordered semigroup (in short DRLsemigroup) if and only if

i) $A=(A,+)$ is a commutative semigroup with zero " 0 ";

ii) $A=(A, \leq)$ is a lattice such that $a+(b \cup c)=(a+b) \cup$ $(a+c)$ and $a+(b \cap c)=(a+b) \cap(a+c)$ for all $a, b, c \in$ $A$

Manuscript received January 30, 2019; accepted April 7, 2020.

Z.T. Wale is with the Department of Mathematics, College of Natural and Computational Sciences, Addis Ababa University, Ethiopia. E-mail: zelalem.teshomedaau.edu.et iii) Given $a, b \in A$, there exists a least $x$ in $\mathrm{A}$ such that $b+x \geq a$, and we denote this $x$ by $a-b$ (for a given $a, b$ this $x$ is uniquely determined);

iv) $(a-b) \cup 0+b \leq a \cup b$ for all $a, b \in A$;

v) $a-a \geq 0$ for all $a \in A$.

Theorem 1: Any DRL-semigroup is a distributive lattice.

Definition 2: A DRL-semigroup $A$ is said to be involutary if there is an element $1(\neq 0)(0$ is the identity w.r.t. +$)$ such that

(i) $a+(1-a)=1+1$;

(ii) $1-(1-a)=a$ for all $a \in \mathrm{A}$.

Theorem 2: In a DRL-semigroup with 1,1 is unique.

Theorem 3: If a DRL-semigroup contains a least element $x$, then $x=0$. Dually, if a DRL-semigroup with 1 contains a largest element $\alpha$, then $\alpha=1$.

Throughout this paper let $I=(I,+,-, \vee, \wedge, 0,1)$ be a dually residuated lattice ordered semigroup satisfying $1-(1-a)=a$ for all $a \in I$.

Lemma 1: Let 1 be the largest element of I. Then for $a, b \in$ I, the following holds

(i) $a+(1-a)=1$;

(ii) $1-a=1-b \Longleftrightarrow a=b$;

(iii) $1-(a \cup b)=(1-a) \cap(1-b)$.

Lemma 2: Let $I$ be complete. If $a_{\alpha} \in I$ for every $\alpha \in \Delta$, then

(i) $1-\bigvee_{\alpha \in \Delta} a_{\alpha}=\bigwedge_{\alpha \in \Delta}\left(1-a_{\alpha}\right)$.

(ii) $1-\bigwedge_{\alpha \in \Delta}^{\alpha \in \Delta} a_{\alpha}=\bigvee_{\alpha \in \Delta}^{\alpha \in \Delta}\left(1-a_{\alpha}\right)$.

Definition 3: An I-vague set $A$ of a non-empty set $W$ is a pair $\left(t_{A}, f_{A}\right)$ where $t_{A}: W \rightarrow I$ and $f_{A}: W \rightarrow I$ with $t_{A}(x) \leq 1-f_{A}(x)$ for all $x \in W$.

Definition 4: The interval $\left[t_{A}(x), 1-f_{A}(x)\right]$ is called the I-vague value of $x \in \mathrm{W}$ and is denoted by $V_{A}(x)$.

Definition 5: Let $B_{1}=\left[a_{1}, b_{1}\right]$ and $B_{2}=\left[a_{2}, b_{2}\right]$ be two I-vague values. We say $B_{1} \geq B_{2}$ if and only if $a_{1} \geq a_{2}$ and $b_{1} \geq b_{2}$.

Definition 6: Let $A=\left(t_{A}, f_{A}\right)$ and $B=\left(t_{B}, f_{B}\right)$ be I-vague sets on a non empty set W. $A$ is said to be contained in $B$ written as $A \subseteq B$ if and only if $t_{A}(x) \leq t_{B}(x)$ and $f_{A}(x) \geq f_{B}(x)$ for all $x \in \mathrm{W}$. A is said to be equal to $\mathrm{B}$ written as $\mathrm{A}=\mathrm{B}$ if and only if $A \subseteq B$ and $B \subseteq A$.

Definition 7: An I-vague set $A$ of $W$ with $V_{A}(x)=V_{A}(y)$ for all $x, y \in W$ is called a constant I-vague set of $W$.

Definition 8: Let A be an I-vague set of a non empty set W. Let $A_{(\alpha, \beta)}=\left\{x \in W: V_{A}(x) \geq[\alpha, \beta]\right\}$ where $\alpha, \beta \in I$ and $\alpha \leq \beta$. Then $A_{(\alpha, \beta)}$ is called the $(\alpha, \beta)$ cut of the I-vague set $\mathrm{A}$. 
Definition 9: Let $\mathrm{S} \subseteq \mathrm{W}$. The characteristic function of $\mathrm{S}$ denoted as $\chi_{S}=\left(t_{\chi_{S}}, f_{\chi_{S}}\right)$, which takes values in I is defined as follows:

$$
t_{\chi_{S}}(x)=\left\{\begin{array}{ll}
1 & \text { if } x \in S \\
0 & \text { otherwise }
\end{array} \quad f_{\chi_{S}}(x)= \begin{cases}0 & \text { if } x \in S \\
1 & \text { otherwise. }\end{cases}\right.
$$

$\chi_{S}$ is called the I-vague characteristic set of S in I. Thus

$$
V_{\chi_{S}}(x)= \begin{cases}{[1,1]} & \text { if } x \in S \\ {[0,0]} & \text { otherwise. }\end{cases}
$$

Definition 10: Let $A=\left(t_{A}, f_{A}\right)$ and $B=\left(t_{B}, f_{B}\right)$ be I-vague sets of a set W.

(i) Their union $A \cup B$ is defined as $A \cup B=\left(t_{A \cup B}, f_{A \cup B}\right)$ where $t_{A \cup B}(x)=t_{A}(x) \vee t_{B}(x)$ and $f_{A \cup B}(x)=f_{A}(x) \wedge f_{B}(x)$ for each $x \in \mathrm{W}$.

(ii) Their intersection $A \cap B$ is defined as $A \cap B=\left(t_{A \cap B}, f_{A \cap B}\right)$ where $t_{A \cap B}(x)=t_{A}(x) \wedge t_{B}(x)$ and $f_{A \cap B}(x)=f_{A}(x) \vee f_{B}(x)$ for each $x \in \mathrm{G}$.

Definition 11: Let $B_{1}=\left[a_{1}, b_{1}\right]$ and $B_{2}=\left[a_{2}, b_{2}\right]$ be I-vague values. Then

(i) $\operatorname{isup}\left\{B_{1}, B_{2}\right\}=\left[\sup \left\{a_{1}, a_{2}\right\}, \sup \left\{b_{1}, b_{2}\right\}\right]$.

(ii) $\operatorname{iinf}\left\{B_{1}, B_{2}\right\}=\left[\inf \left\{a_{1}, a_{2}\right\}, \inf \left\{b_{1}, b_{2}\right\}\right]$.

Lemma 3: Let $A$ and $B$ be I-vague sets of a set $W$. Then $A \cup B$ and $A \cap B$ are also I-vague sets of $W$.

Let $x \in \mathrm{W}$. From the definition of $A \cup B$ and $A \cap B$ we have

(i) $V_{A \cup B}(x)=\operatorname{isup}\left\{V_{A}(x), V_{B}(x)\right\}$;

(ii) $V_{A \cap B}(x)=\operatorname{iinf}\left\{V_{A}(x), V_{B}(x)\right\}$.

Definition 12: Let I be complete and $\left\{A_{i}=\left(t_{A_{i}}, f_{A_{i}}\right): i \in \triangle\right\}$ be a non empty family of I vague sets of $\mathrm{W}$. Then for each $x \in W$,

(i) $\operatorname{isup}\left\{V_{A_{i}}(x): i \in \triangle\right\}=\left[\bigvee_{i \in \triangle} t_{A_{i}}(x), \bigvee_{i \in \triangle}\left(1-f_{A_{i}}\right)(x)\right]$

(i) $\operatorname{iinf}\left\{V_{A_{i}}(x): i \in \triangle\right\}=\left[\bigwedge_{i \in \triangle} t_{A_{i}}(x), \bigwedge_{i \in \triangle}\left(1-f_{A_{i}}\right)(x)\right]$.

Lemma 4: Let I be complete. If $\left\{A_{i}: i \in \triangle\right\}$ is a non empty family of I-vague sets of $\mathrm{W}$, then $\bigcup A_{i}$ and $\cap A_{i}$ are also an I-vague sets of W.

Definition 13: Let I be complete and $\left\{A_{i}=\left(t_{A_{i}}, f_{A_{i}}\right): i \in \triangle\right\}$ be a non empty family of I vague sets of $\mathrm{W}$. Then for each $x \in W$,

(i) $\operatorname{isup}\left\{V_{A_{i}}(x): i \in \triangle\right\}=\left[\bigvee_{i \in \triangle} t_{A_{i}}(x), \bigvee_{i \in \triangle}\left(1-f_{A_{i}}\right)(x)\right]$.

(ii) $\operatorname{iinf}\left\{V_{A_{i}}(x): i \in \triangle\right\}=\left[\bigwedge_{i \in \triangle} t_{A_{i}}(x), \bigwedge_{i \in \triangle}\left(1-f_{A_{i}}\right)(x)\right]$.

Definition 14: Let $\Phi: X \rightarrow Y$ be a mapping from a set $X$ into a set $Y$. Let $B$ be an I-vague set of $Y$. Then the preimage of $B, \Phi^{-1}(B)=\left(t_{\Phi^{-1}(B)}, f_{\Phi^{-1}(B)}\right)$ is given by $t_{\Phi^{-1}(B)}: X \rightarrow I$ and $f_{\Phi^{-1}(B)}: X \rightarrow I$ where $t_{\Phi^{-1}(B)}(x)=t_{B}(\Phi(x))$ and $f_{\Phi^{-1}(B)}(x)=$ $t_{B}(\Phi(x))$ for each $x \in X$.

Lemma 5: Let $\Phi: X \rightarrow Y$ be a mapping from a set $X$ into a set $Y$. If $B$ be an I-vague set of $Y$, then $\Phi^{-1}(B)$ is an I-vague set of $X$ and $V_{\Phi^{-1}(B)}(x)=V_{B} \Phi(x)$ for each $x \in X$.

Definition 15: Let $I$ be complete and $\Phi: X \rightarrow Y$ be a mapping from a set $X$ into a set $Y$. Let $\mathrm{A}=\left(t_{A}, f_{A}\right)$ be an I-vague set of $X$. Then the image of $A, \Phi(A)=\left(t_{\Phi(A)}, f_{\Phi(A)}\right)$ is given by

$$
t_{\Phi(A)}(y)= \begin{cases}\bigvee_{x \in \Phi^{-1}(y)} t_{A}(z) & \text { if } \Phi^{-1}(y) \neq \emptyset \\ 0 & \text { otherwise }\end{cases}
$$

$$
f_{\Phi(A)}(y)= \begin{cases}\bigwedge_{x \in \Phi^{-1}(y)} t_{A}(z) & \text { if } \Phi^{-1}(y) \neq \emptyset \\ 1 & \text { otherwise. }\end{cases}
$$

Lemma 6: Let $I$ be complete and $\Phi: X \rightarrow Y$ be a mapping from a set $X$ into a set $Y$. If $A$ be an I-vague set of $X$, then $\Phi(A)$ is an I-vague set of $Y$.

Theorem 4: Let $I$ be complete and $\Phi: X \rightarrow Y$ be a mapping from a set $X$ into a set $Y$. If $A$ is an I-vague set of $X$, then

$$
V_{\Phi(A)}(y)= \begin{cases}\operatorname{isup}\left\{V_{A}(z): z \in \Phi^{-1}(y)\right\} & \text { if } \Phi^{-1}(y) \neq \emptyset \\ {[0,0]} & \text { otherwise. }\end{cases}
$$

\section{I-VAGUE Vector SPACES}

Definition 16: Let $W$ be a vector space over a field $F$ and $A$ be an I-vague set of $W$. Then $A$ is said to be an I-vague subspace of $W$ if

(i) $V_{A}(x+y) \geq \operatorname{iinf}\left\{V_{A}(x), V_{A}(y)\right\}$

(ii) $V_{A}(\lambda x) \geq V_{A}(x)$ for all $x, y \in \mathrm{W}$ and $\lambda \in F$

Example 1: Let $I$ be the unit interval $[0,1]$ of real numbers. Let $a \oplus b=\min \{1, a+b\}$. with the usual ordering $(I, \oplus, \leq,-)$ is an involutary DRL-semigroup. Consider the vector space $W=\Re^{2}$ over $\mathfrak{R}$. Let $A=\left(t_{A}, f_{A}\right)$ where $t_{A}: \Re^{2} \rightarrow[0,1]$ by $t_{A}(x, y)=1$ and $f_{A}: \mathfrak{R}^{2} \rightarrow[0,1]$ by $f_{A}(x, y)=0$ for all $(x, y) \in \mathfrak{R}^{2}$. Then $\mathrm{A}$ is an I-vague subspace of $\mathrm{W}$.

Lemma 7: Let $A$ be an I-vague subspace of $W$. Then

(i) $V_{A}(0) \geq V_{A}(x)$ for all $x \in \mathrm{W}$.

(ii) $V_{A}(\lambda x)=V_{A}(x)$ for all $x \in \mathrm{W}$ and $\lambda \neq 0$.

Proof: Let $A$ be an I-vague subspace of $W$.

(i) $V_{A}(0) \doteq V_{A}(0 x) \geq V_{A}(x)$. Hence $V_{A}(0) \geq V_{A}(x)$ for all $x \in \mathrm{W}$.

(ii) Let $\lambda \neq 0$ and $x \in W$. Then $V_{A}(x)=V_{A}\left(\left(\lambda^{-1} \lambda\right) x\right)=$ $V_{A}\left(\left(\lambda^{-1}\right)(\lambda x)\right) \geq V_{A}(\lambda x) \geq V_{A}(x)$.

Hence $V_{A}(\lambda x)=V_{A}(x)$ for all $\lambda \in F \backslash\{0\}$.

Lemma 8: Let $W$ be a vector space over a field $F$. A is an I-vague subspace of $W$ iff $V_{A}(\lambda x+\mu y) \geq \operatorname{iinf}\left\{V_{A}(x), V_{A}(y)\right\}$ for all $\lambda, \mu \in F$ and $x, y \in W$.

Proof: Let $A$ be an I-vague subspace of $W$. Let $x, y \in \mathrm{W}$ and $\lambda, \mu \in \mathrm{F}$. Then $V_{A}(\lambda x) \geq V_{A}(x)$ and $V_{A}(\mu y) \geq V_{A}(y)$. Since $\mathrm{A}$ is an I-vague subspace of $\mathrm{W}, \quad V_{A}(\lambda x+\mu y) \geq \operatorname{iinf}\left\{V_{A}(\lambda x), V_{A}(\mu y)\right\}$. Moreover $\operatorname{iinf}\left\{V_{A}(\lambda x), V_{A}(\mu y)\right\} \geq \operatorname{iinf}\left\{V_{A}(x), V_{A}(y)\right\}$. Hence $V_{A}(\lambda x+\mu y) \geq \operatorname{iinf}\left\{V_{A}(x), V_{A}(y)\right\}$. Conversely, suppose that $V_{A}(\lambda x+\mu y) \geq \operatorname{iinf}\left\{V_{A}(x), V_{A}(y)\right\}$ for all $\lambda, \mu \in F$ and $x, y \in W$. Put $\lambda=\mu=1$. Then $V_{A}(x+y) \geq \operatorname{iinf}\left\{V_{A}(x), V_{A}(y)\right\}$. Moreover, $V_{A}(\lambda x)=V_{A}(\lambda x+0 x) \geq \operatorname{iinf}\left\{V_{A}(x), V_{A}(x)\right\}=V_{A}(x)$. This proves the lemma.

Moreover, $V_{A}(x-y)=V_{A}(x+-1 y) \geq \operatorname{iinf}\left\{V_{A}(x), V_{A}(y)\right\}$.

Lemma 9: Let $W$ be a vector space over a field $F$ and $A$ be an I-vague subspace of $W$. Then $V_{A}\left(\lambda_{1} x_{1}+\lambda_{2} x_{2}+\ldots+\lambda_{n} x_{n}\right) \geq$ $\operatorname{iinf}\left\{V_{A}\left(x_{1}\right), V_{A}\left(x_{2}+\ldots+V_{A}\left(x_{n}\right)\right\}\right.$ for all $\lambda_{1}, \lambda_{2}, \ldots, \lambda_{n} \in F$ and $x_{1}, x_{2}, \ldots, x_{n} \in W$.

Proof: We use proof by induction. Clearly the statement is true for $n=2$. Assume that the statement is true for $n$.

$$
\begin{aligned}
& V_{A}\left(\lambda_{1} x_{1}+\lambda_{2} x_{2}+\ldots+\lambda_{n} x_{n}+\lambda_{n+1} x_{n+1}\right) \\
& =V_{A}\left(\left(\lambda_{1} x_{1}+\lambda_{2} x_{2}+\ldots+\lambda_{n} x_{n}\right)+\lambda_{n+1} x_{n+1}\right)
\end{aligned}
$$




$$
\begin{aligned}
& \geq \operatorname{iinf}\left\{V_{A}\left(\lambda_{1} x_{1}+\lambda_{2} x_{2}+\ldots+\lambda_{n} x_{n}\right), V_{A}\left(\lambda_{n+1} x_{n+1}\right)\right\} \\
& \geq \operatorname{iinf}\left\{\operatorname{iinf}\left\{V_{A}\left(x_{1}\right), V_{A}\left(x_{2}\right), \ldots, V_{A}\left(x_{n}\right)\right\}, V_{A}\left(x_{n+1}\right)\right\} \\
& =\operatorname{iinf}\left\{V_{A}\left(x_{1}\right), V_{A}\left(x_{2}\right), \ldots, V_{A}\left(x_{n}\right), V_{A}\left(x_{n+1}\right)\right\}
\end{aligned}
$$

Therefore $V_{A}\left(\lambda_{1} x_{1}+\lambda_{2} x_{2}+\ldots+\lambda_{n} x_{n}+\lambda_{n+1} x_{n+1}\right) \geq$ $\operatorname{iinf}\left\{V_{A}\left(x_{1}\right), V_{A}\left(x_{2}\right), \ldots, V_{A}\left(x_{n}\right), V_{A}\left(x_{n+1}\right)\right\}$. Hence the lemma follows.

Theorem 5: An I-vague set $A$ of a vector space $W$ is an I-vague subspace of $\mathrm{W}$ iff for all $\alpha, \beta \in I$ with $\alpha \leq \beta$, the I-vague cut $A_{(\alpha, \beta)}$ of $W$ whenever it is non empty.

Proof: Let $A$ be an I-vague set of a vector space $W$. Suppose that $A$ is an I-vague subspace of $W$. We prove that $A_{(\alpha, \beta)}$ is a subspace of $\mathrm{W}$ whenever it is non empty. Let $x, y \in A_{(\alpha, \beta)}$. Then $V_{A}(x) \geq[\alpha, \beta]$ and $V_{A}(y) \geq[\alpha, \beta]$. It follows that $\operatorname{iinf}\left\{V_{A}(x), V_{A}(y)\right\} \geq[\alpha, \beta]$. Since $V_{A}(x+y) \geq$ $\operatorname{iinf}\left\{V_{A}(x), V_{A}(y)\right\}, V_{A}(x+y) \geq[\alpha, \beta]$. Hence $x+y \in A_{(\alpha, \beta)}$. Let $x \in A_{(\alpha, \beta)}$ and $\lambda \in F$. Then $V_{A}(\lambda x) \geq V_{A}(x) \geq[\alpha, \beta]$. Hence $\lambda x \in A_{(\alpha, \beta)}$. Therefore $A_{(\alpha, \beta)}$ is a subspace of $W$.

Conversely, suppose that $A_{(\alpha, \beta)}$ is a subspace of $W$ whenever it is non empty. We prove that $A$ is an I-vague subspace of $W$. Let $x, y \in W$. Suppose $V_{A}(x)=[\alpha, \beta]$ and $V_{A}(y)=[\gamma, \delta]$ for some $\alpha, \beta, \gamma, \delta \in I . \operatorname{iinf}\left\{V_{A}(x), V_{A}(y)\right\}=[\alpha \wedge \gamma, \beta \wedge \delta]=$ $[\xi, \eta]$ for some $\xi, \eta \in I$. Hence $x, y \in A_{(\xi, \eta)}$. Since $A_{(\xi, \eta)}$ is a subspace of $W, \lambda x+\mu y \in A_{(\xi, \eta)}$ for $\lambda, \mu \in F$. Hence $V_{A}(\lambda x+\mu y) \geq[\xi, \eta]=\operatorname{iinf}\left\{V_{A}(x), V_{A}(y)\right\}$. Thus, $V_{A}(\lambda x+$ $\mu y) \geq \operatorname{iinf}\left\{V_{A}(x), V_{A}(y)\right\}$. Hence the theorem follows.

Lemma 10: Let $A$ be an I-vague subspace of a vector space $W$. Then the set $W_{A}=\left\{x \in W: V_{A}(x)=V_{A}(0)\right\}$ is a subspace of $W$.

Proof: Since $0 \in W_{A}, W_{A} \neq \emptyset$. Let $x, y \in W_{A}$. Then $V_{A}(x)=$ $V_{A}(y)=V_{A}(0)$. Hence $V_{A}(x+y) \geq \operatorname{iinf}\left\{V_{A}(x), V_{A}(y)\right\}=V_{A}(0)$. Since $V_{A}(0) \geq V_{A}(x+y), V_{A}(x+y)=V_{A}(0)$. Hence $x+y \in W_{A}$. Let $\lambda \in \mathrm{F}$ and $x \in W_{A}$. Then $V_{A}(x)=V_{A}(0) . V_{A}(\lambda x) \geq V_{A}(x)=$ $V_{A}(0)$. Thus $V_{A}(\lambda x)=V_{A}(0)$. Hence $\lambda x \in W_{A}$. Therefore $W_{A}$ is a subspace of $\mathrm{W}$.

Lemma 11: Let $U$ be a subspace of a vector space $\mathrm{W}$ with $\alpha, \beta, \gamma, \delta \in I, \alpha \leq \beta, \gamma \leq \delta$ and $[\gamma, \delta] \leq[\alpha, \beta]$. Then the I-vague set $A$ of $W$ defined by

$$
V_{A}(x)= \begin{cases}{[\alpha, \beta]} & \text { if } x \in U \\ {[\gamma, \delta]} & \text { otherwise }\end{cases}
$$

is an I-vague subspace of $W$.

Proof: Let $U$ be a subspace of $W$. We have the following three cases:

(i) Let $x, y \in U$. Since $U$ is a subspace of $W, \lambda x+\mu y \in U$ for $\lambda, \mu \in F . V_{A}(\lambda x+\mu y)=[\alpha, \beta]=\operatorname{iinf}\left\{V_{A}(x), V_{A}(y)\right\}$. It follows that $V_{A}(\lambda x+\mu y) \geq \operatorname{iinf}\left\{V_{A}(x), V_{A}(y)\right\}$.

(ii) Exactly one of $x$ or $y$ does not belong to $U$. Suppose $x \in U$ and $y \notin U . \lambda x+\mu y \notin U$ for any $\mu \neq 0 . V_{A}(\lambda x+$ $\mu y)=[\gamma, \delta]=\operatorname{iinf}\left\{V_{A}(x), V_{A}(y)\right\}$. Hence $V_{A}(\lambda x+\mu y) \geq$ $\operatorname{iinf}\left\{V_{A}(x), V_{A}(y)\right\}$.

(iii) Both $x$ and $y$ does not belong to $U$. $\lambda x+\mu y \notin U$ for any $\lambda, \mu, \neq 0$. iinf $\left\{V_{A}(x), V_{A}(y)\right\}=[\gamma, \delta]=V_{A}(\lambda x+\mu y)$. Hence $V_{A}(\lambda x+\mu y) \geq \operatorname{iinf}\left\{V_{A}(x), V_{A}(y)\right\}$.

This proves the lemma.

Lemma 12: Let $A$ and $B$ be I-vague subspaces of a vector space $W$. Then $A \cap B$ is also I-vague subspace of $W$.
Proof: Let $A$ and $B$ be I-vague subspaces of $W$. We prove that $A \cap B$ is also an I-vague subspace of $W$. By Lemma $3, A \cap B$ is an I-vague set of $W$. Let $x, y \in \mathrm{W}$.

$$
\begin{aligned}
V_{A \cap B}(x+y) & =\operatorname{iinf}\left\{V_{A}(x+y), V_{B}(x+y)\right\} \\
& \geq \operatorname{iinf}\left\{\operatorname{iinf}\left\{V_{A}(x), V_{A}(y)\right\}, \operatorname{iinf}\left\{V_{B}(x), V_{B}(y)\right\}\right\} \\
& =\operatorname{iinf}\left\{\operatorname { i i n f } \left\{\left\{V_{A}(x), V_{B}(x)\right\}, \operatorname{iinf}\left\{\left\{V_{A}(y), V_{B}(y)\right\}\right\}\right.\right. \\
& =\operatorname{iinf}\left\{V_{A \cap B}(x), V_{A \cap B}(y)\right\}
\end{aligned}
$$

Hence $V_{A \cap B}(x+y) \geq \operatorname{iinf}\left\{V_{A \cap B}(x), V_{A \cap B}(y)\right\} . \quad V_{A \cap B}(\lambda x)=$ iinf $\left\{V_{A}(\lambda x), V_{B}(\lambda x)\right\} \geq \operatorname{iinf}\left\{V_{A}(x), V_{B}(x)\right\}=V_{A \cap B}(x)$. Thus $V_{A \cap B}(\lambda x) \geq V_{A \cap B}(x)$. Therefore $A \cap B$ is an I-vague subspace of $W$.

Lemma 13: Let $I$ be complete. If $\left\{A_{i}: i \in \triangle\right\}$ is a non empty family of I-vague subspaces of $W$, then $\bigcap A_{i}$ is an I-vague subspace of $W$.

Proof: Let $\left\{A_{i}: i \in \triangle\right\}$ be a non empty family of I-vague subspaces of $W$. Let $A=\bigcap_{i \in \triangle} A_{i}$. We prove that $A$ is an I-vague subspace of $W$. By Lemma $4, A$ is an I-vague set of W.Let $x, y \in W$. Then

$$
\begin{aligned}
V_{A}(x+y) & =\operatorname{iinf}\left\{V_{A_{i}}(x+y): i \in \triangle\right\} \\
& \geq \operatorname{iinf}\left\{\operatorname{iinf}\left\{V_{A_{i}}(x), V_{A_{i}}(y)\right\}: i \in \triangle\right\} \\
& =\operatorname{iinf}\left\{\operatorname{iinf}\left\{V_{A_{i}}(x): i \in \triangle\right\}, \operatorname{iinf}\left\{V_{A_{i}}(y): i \in \triangle\right\}\right\} \\
& =\operatorname{iinf}\left\{V_{A}(x), V_{A}(y)\right\} .
\end{aligned}
$$

Thus $V_{A}(x+y) \geq \operatorname{iinf}\left\{V_{A}(x), V_{A}(y)\right\} \cdot V_{A}(\lambda x)=\operatorname{iinf}\left\{V_{A_{i}}(\lambda x):\right.$ $\left.i \in \triangle\}\} \geq \operatorname{iinf}\left\{V_{A_{i}}(x): i \in \triangle\right\}\right\}=V_{A}(x)$. Hence the lemma follows.

Example 2: Consider $W=\mathfrak{R}^{2}$ over $\mathfrak{R}$.Then $W_{1}=\{(x, y)$ : $x+y=0\}$

and $W_{2}=\{(x, y): x-y=0\}$ are subspaces of $W$.

$V_{A}(x)=\left\{\begin{array}{ll}{[\alpha, \beta]} & \text { if } x \in W_{1} \\ {[\gamma, \delta]} & \text { otherwise. }\end{array} \quad V_{B}(x)= \begin{cases}{[\alpha, \beta]} & \text { if } x \in W_{2} \\ {[\gamma, \delta]} & \text { otherwise }\end{cases}\right.$

with $\alpha, \beta, \gamma, \delta \in I, \alpha \leq \beta, \gamma \leq \delta$ and $[\alpha, \beta] \leq[\gamma, \delta]$. We show that $A \cup B$ is not an I-vague subspace of $W$. Let $\mathrm{u}=(1,-1)$ and $\mathrm{v}=(1,1)$.

$$
\begin{gathered}
V_{A \cup B}(u+v)=V_{A \cup B}(2,0)=\operatorname{isup}\left\{V_{A}(2,0), V_{B}(2,0)=[\gamma, \delta] .\right. \\
V_{A \cup B}(u)=V_{A \cup B}(1,-1)=\operatorname{isup}\left\{V_{A}(1,-1), V_{B}(1,-1)=[\alpha, \beta] .\right. \\
V_{A \cup B}(v)=V_{A \cup B}(1,1)=\operatorname{isup}\left\{V_{A}(1,1), V_{B}(1,1)\right\}=[\alpha, \beta] . \\
\operatorname{iinf}\left\{V_{A \cup B}(u), V_{A \cup B}(v)\right\}=[\alpha, \beta] . \\
V_{A \cup B}(u+v)=[\gamma, \delta] \nsupseteq[\alpha, \beta]=\operatorname{iinf}\left\{V_{A \cup B}(u), V_{A \cup B}(v)\right\} .
\end{gathered}
$$

Therefore $A \cup B$ is not an I-vague subspace of $W$.

Lemma 14: Let $U \neq \emptyset$. The I-vague characteristic function set of $U, \chi_{U}$ is an I-vague subspace of $W$ iff $U$ is a subspace of $W$.

Proof: Suppose that $\chi_{U}$ is an I-vague subspace of $W$. Let $x, y \in \mathrm{U}$. Then $V_{\chi_{U}}(x)=[1,1]$ and $V_{\chi_{U}}(y)=[1,1]$. Since $\chi_{U}$ is an I-vague subspace of $W, V_{\chi_{U}}(x+y) \geq$ $\operatorname{iinf}\left\{V_{\chi_{U}}(x), V_{\chi_{U}}(y)\right\}=[1,1]$. Hence $V_{\chi_{U}}(x+y)=[1,1]$. So, $x+y \in U$. $V_{\chi_{U}}(\lambda x) \geq V_{\chi_{U}}(x)=[1,1]$. It follows that $V_{\chi_{U}}(\lambda x)=[1,1]$. Hence $\lambda x \in U$. Therefore $U$ is a subspace 
of $W$. Conversely, suppose that $U$ is a subspace of $W$. Then Consider

$$
V_{\chi_{U}}(x)= \begin{cases}{[1,1]} & \text { if } x \in U \\ {[0,0]} & \text { otherwise }\end{cases}
$$

By Lemma 11, $\chi_{U}$ is an I-vague subspace of $W$.

Theorem 6: Let $A$ be an I-vague subspace of a vector space $W$. If $V_{A}(x-y)=V_{A}(0)$ for all $x, y \in W$, then $V_{A}(x)=V_{A}(y)$.

Proof: Let $A$ be an I-vague subspace of a vector space $W$. Suppose that $V_{A}(x-y)=V_{A}(0)$ for $x, y \in W$. We prove that $V_{A}(x)=V_{A}(y) . V_{A}(x-y)=V_{A}(0)$ implies that $V_{A}(y-x)=$ $V_{A}(0)$.

$$
\begin{aligned}
V_{A}(x) & =V_{A}((x-y)+y) . \\
& \geq \operatorname{iinf}\left\{V_{A}(x-y), V_{A}(y)\right\} \\
& =\operatorname{iinf}\left\{V_{A}(0), V_{A}(y)\right\} \\
& =V_{A}(y)
\end{aligned}
$$

Thus $V_{A}(x) \geq V_{A}(y)$. Similarly, $\quad V_{A}(y)=V_{A}((y-x)+$ $x) \geq \operatorname{iinf}\left\{V_{A}(y-x), V_{A}(x)\right\}=\operatorname{iinf}\left\{V_{A}(0), V_{A}(x)\right\}=V_{A}(x)$. Thus $V_{A}(y) \geq V_{A}(x)$. Hence $V_{A}(x)=V_{A}(y)$.

The following example shows that the converse of the above theorem is not true.

Example 3: Let $I$ be the unit interval $[0,1]$ of real numbers. Define $a \oplus b=\min \{1, a+b\}$. With the usual ordering $(I, \oplus, \leq$ $,-)$ is an involutary DRL-semigroup. Let $W=\mathfrak{R}^{2}$ over $\mathfrak{R}$. Then $U=\{(x, y): x+2 y=0\}$ is a subspace of $W$. Define the I-vague subspace $A$ of $W$ by

$$
V_{A}(u)= \begin{cases}{\left[\frac{1}{2}, 1\right]} & \text { if } u \in U \\ {\left[0, \frac{1}{4}\right]} & \text { otherwise. }\end{cases}
$$

Let $u=(-2,2)$ and $v=(1,2) . V_{A}(u)=V_{A}(v)=\left[0, \frac{1}{4}\right]$ and $V_{A}(u-v)=V_{A}(-3,0)=\left[0, \frac{1}{4}\right] \neq V_{A}(0)$. Thus $V_{A}(u)=V_{A}(v)$ but $V_{A}(u-v) \neq V_{A}(0)$.

Theorem 7: Let $A$ be an I-vague subspace of a vector space $W$ and $x \in W$. Then $V_{A}(x+y)=V_{A}(y)$ for all $y \in W$ iff $V_{A}(x)=$ $V_{A}(0)$.

Proof: Let $A$ be an I-vague subspace of a vector space $W$ and $x \in W$. Suppose that $V_{A}(x+y)=V_{A}(y)$ for all $y \in W$. Take $y=0$. Hence $V_{A}(x)=V_{A}(0)$. Conversely, suppose that $V_{A}(x)=$ $V_{A}(0)$. Let $y \in W$. Then $V_{A}(x+y) \geq \operatorname{iinf}\left\{V_{A}(x), V_{A}(y)\right\}=V_{A}(y)$. It follows that $V_{A}(x+y) \geq V_{A}(y)$.

$$
\begin{aligned}
V_{A}(y) & =V_{A}(-x+x+y) \\
& \geq \operatorname{iinf}\left\{V_{A}(-x), V_{A}(x+y)\right\} \\
& =\operatorname{iinf}\left\{V_{A}(x), V_{A}(x+y)\right\} \\
& =\operatorname{iinf}\left\{V_{A}(0), V_{A}(x+y)\right\} \\
& =V_{A}(x+y)
\end{aligned}
$$

Thus $V_{A}(y) \geq V_{A}(x+y)$. It follows that $V_{A}(x+y)=V_{A}(y)$.

Theorem 8: Let $A$ be an I-vague subspace of a vector space $W$. If $V_{A}(x-y)=V_{A}(0)$ for all $x, y \in W$, then $V_{A}(x)=V_{A}(y)$.

Proof: Let $A$ be an I-vague subspace of a vector space $W . V_{A}(x)=V_{A}((x-y)+y) \geq \operatorname{iinf}\left\{V_{A}(x-y), V_{A}(y)\right\}=$ $\operatorname{iinf}\left\{V_{A}(0), V_{A}(y)\right\}=V_{A}(y)$. Similarly, $V_{A}(y)=V_{A}((y-x)+$ $x) \geq \operatorname{iinf}\left\{V_{A}(y-x), V_{A}(x)\right\}=\operatorname{iinf}\left\{V_{A}(0), V_{A}(x)\right\}=V_{A}(x)$. Hence $V_{A}(x)=V_{A}(y)$.

Theorem 9: Let $W_{1}$ and $W_{2}$ be vector spaces over a field $F$, and let $T$ be a linear transformation from $W_{1}$ into $W_{2}$. If
$A$ is an I-vague subspace of $W_{2}$, then $T^{-1}(A)$ is an I-vague subspace of $W_{1}$.

Proof: Let $T$ be a linear transformation from $W_{1}$ into $W_{2}$ and $A$ be an I-vague subspace of $W_{2}$.

$$
\begin{aligned}
V_{T^{-1}(A)}(\lambda x+\mu y) & =V_{A}(T(\lambda x+\mu y)) \\
& =V_{A}(\lambda T(x)+\mu T(y)) \\
& \geq \operatorname{iinf}\left\{V_{A}(\lambda T(x)), V_{A}(\mu T(y))\right\} \\
& \geq \operatorname{iinf}\left\{V_{A}(T(x)), V_{A}(T(y))\right\} \\
& =\operatorname{iinf}\left\{V_{T^{-1}(A)}(x), V_{T^{-1}(A)}(y)\right\}
\end{aligned}
$$

This completes the proof.

Theorem 10: Let $I$ be complete and infinitely meet distributive. Let $U$ and $V$ be vector spaces over a field $F$ and $T: U \rightarrow V$ be a linear transformation. If $A$ is an I-vague subspace of $U$, then $T(A)$ is an I-vague subspace of $V$.

Proof: Let $T: U \rightarrow V$ be a linear transformation and $A$ be an I-vague subspace of $U$.

$$
\begin{aligned}
V_{T(A)}\left(y_{1}+y_{2}\right)= & \operatorname{isup}\left\{V_{A}(z): z \in T^{-1}\left(y_{1}+y_{2}\right)\right\} \\
\geq & \operatorname{isup}\left\{V_{A}(z): z=x_{1}+x_{2} \text { where } x_{1} \in T^{-1}\left(y_{1}\right)\right. \\
& \left.\quad \text { and } x_{2} \in T^{-1}\left(y_{2}\right)\right\} \\
= & \operatorname{isup}\left\{V_{A}\left(x_{1}+x_{2}\right): x_{1} \in T^{-1}\left(y_{1}\right)\right. \text { and } \\
\quad & \left.x_{2} \in T^{-1}\left(y_{2}\right)\right\} \\
\geq & \operatorname{isup}\left\{\operatorname{iinf}\left\{V_{A}\left(x_{1}\right), V_{A}\left(x_{2}\right)\right\}: x_{1} \in T^{-1}\left(y_{1}\right)\right. \\
\quad & \left.\quad \operatorname{and} x_{2} \in T^{-1}\left(y_{2}\right)\right\} \\
= & \operatorname{iinf}\left\{\operatorname{isup}\left\{V_{A}\left(x_{1}\right), V_{A}\left(x_{2}\right)\right\}: x_{1} \in T^{-1}\left(y_{1}\right)\right. \\
& \text { and } \left.x_{2} \in T^{-1}\left(y_{2}\right)\right\}
\end{aligned}
$$

since $I$ is infinitely meet distributive

$$
=\operatorname{iinf}\left\{V_{T(A)}\left(y_{1}\right), V_{T(A)}\left(y_{2}\right)\right\}
$$

$$
\begin{aligned}
V_{T(A)}(y) & =\operatorname{isup}\left\{V_{A}(z): z \in T^{-1}(y)\right\} \\
& =\operatorname{isup}\left\{V_{A}(z): T(z)=(y)\right\} \\
& \leq \operatorname{isup}\left\{V_{A}(\lambda z): T(z)=y \text { for any } \lambda \in F\right\} \\
& =\operatorname{isup}\left\{V_{A}(\lambda z): T(\lambda z)=\lambda y\right\} \\
& =\operatorname{isup}\left\{V_{A}(u): T(u)=\lambda y\right\} \\
& =V_{T(A)}(\lambda y)
\end{aligned}
$$

This proves the theorem.

\section{REFERENCES}

[1] N. Ramakrishna and T. Eswarlal, "Boolean vague sets," International Journal of Computational Cognition, vol. 5, no. 4, 2007.

[2] K. Swamy, "Dually residuated lattice ordered semigroups," Mathematische Annalen, vol. 159, no. 2, pp. 105-114, 1965.

[3] _ , "Dually residuated lattice ordered semigroups, ii," Mathematische Annalen, vol. 160, no. 1, pp. 64-71, 1965.

[4] — "Dually residuated lattice ordered semigroups, iii," Mathematische Annalen, vol. 167, no. 1, pp. 71-74, 1966.

[5] C. Chang, "Algebraic analysis of many valued logics," Transactions of the American Mathematical society, vol. 88, no. 2, pp. 467-490, 1958.

[6] T. Eswarlal and N. Ramakrishna, "Vague fields and vague vector spaces," International Journal of pure and applied Mathematics, vol. 94, no. 3, pp. 295-305, 2014.

[7] K. R. Rao, "Vague vector space and vague modules," International Journal of Pure and Applied Mathematics, vol. 111, no. 2, pp. 179-188, 2016.

[8] T. Zelalem, "I-vague sets and I-vague relations," International Journal of Computational Cognition, vol. 8, no. 4, pp. 102-109, 2010. 